\title{
Unsteady boundary-layer flow and heat transfer of a nanofluid over a permeable stretching/shrinking sheet.
}

\begin{abstract}
The unsteady boundary layer flow of a nanofluid over a permeable stretching/shrinking sheet is theoretically studied. The governing partial differential equations are transformed into ordinary ones using a similarity transformation, before being solved numerically. The results are obtained for the skin friction coefficient, the local Nusselt number and the local Sherwood number as well as the velocity, temperature and the nanoparticle fraction profiles for some values of the governing parameters, namely, the unsteadiness parameter, the mass suction parameter, the Brownian motion parameter, the thermophoresis parameter, Prandtl number, Lewis number and the stretching/shrinking parameter. It is found that dual solutions exist for both stretching and shrinking cases. The results also indicate that both unsteadiness and mass suction widen the range of the stretching/shrinking parameter for which the solution exists.
\end{abstract}

Keyword: Nanofluid; Stretching/shrinking sheet; Unsteady boundary layer; Dual solutions. 